\title{
Influence of Intraoperative Capsule Rupture on Outcomes in Stage I Epithelial Ovarian Cancer
}

\author{
Jamie N. Bakkum-Gamez, MD, Debra L. Richardson, MD, Leigh G. Seamon, Do, \\ Giovanni D. Aletti, MD, Cecelia A. Powless, MD, Gary L. Keeney, MD, David M. O'Malley, MD, \\ and William A. Cliby, MD
}

OBJECTIVE: To evaluate the effect of tumor capsule rupture on disease prognosis in stage I epithelial ovarian cancer.

METHODS: All patients with International Federation of Gynecology and Obstetrics stage I epithelial ovarian cancer operated on at the Mayo Clinic and The Ohio State University between January 1991 and December 2007 were identified. Relevant tumor characteristics, procedures performed, adjuvant therapies, and follow-up were recorded and analyzed. Inclusion criteria included comprehensive staging. Cox proportional hazards, Kaplan-Meier estimation, log rank test, and $\chi^{2}$ test were used for statistical analyses.

RESULTS: There were 161 cases that met inclusion criteria. Seventy-four (46\%) patients had intact capsules without positive cytology or surface involvement; 61 (38\%) had capsule rupture; $33(20 \%)$ had positive cytology; and $22(14 \%)$ had surface involvement. Overall, 22 of 161 (14\%) patients recurred and 12 of $161(7 \%)$ patients died of their disease. In univariable analysis, both intraoperative capsule rupture and positive cytologic washings portended worse disease-free survival (hazard ratio [HR] 3.6, 95\% confidence interval $[\mathrm{CI}] 1.5-8.9 ; P=.004$ and $\mathrm{HR}$ 5.2, 95\% Cl 2.1-12.3; $P<.001$, respectively) and disease-

See related editorial on page 4 .

From the Division of Gynecologic Oncology, Mayo Clinic, Rochester, Minnesota; Division of Gynecologic Oncology, the Ohio State University College of Medicine, Columbus; and Department of Laboratory Medicine and Pathology, Mayo Clinic, Rochester, Minnesota.

Oral presentation at the Society of Gynecologic Oncologists Winter Meeting, Park City, Utah, February 15-17, 2008.

Corresponding author: Jamie N. Bakkum-Gamez, MD, 201 1st Street SW, Rochester, MN 55905; e-mail: bakkum.jamie@mayo.edu.

Financial Disclosure

The authors did not report any potential conflicts of interest.

(C) 2008 by The American College of Obstetricians and Gynecologists. Published by Lippincott Williams \& Wilkins.

ISSN: 0029-7844/08 specific survival (HR 4.1, 95\% CI 1.3-15.4; $P=.018$ and $\mathrm{HR}$ 5.9, 95\% Cl 1.8-19.3; $P=.005$, respectively). In multivariable analysis, capsule rupture (HR 4.2, 95\% CI 1.8-10.9; $P=.001)$ and positive cytologic washings (HR 6.4, 95\% Cl 2.5-16.0; $P<.001)$ remained independent predictors of worse disease-free survival. Disease-free survival and disease-specific survival were shortest for stage IC cases with positive cytology, surface involvement, or both, that also had intraoperative rupture.

CONCLUSION: In stage I epithelial ovarian cancer, intraoperative capsule rupture portends a higher risk of disease recurrence and death from disease. Careful intraoperative removal of ovarian masses is important, and recognizing the higher-risk nature of such cases is imperative.

(Obstet Gynecol 2009;113:11-7)

LEVEL OF EVIDENCE: III

Epithelial ovarian cancer is the second most com- mon gynecologic malignancy in the United States. In 2008, an estimated 21,650 new cases will be diagnosed and an estimated 15,520 women will die of the disease, making it the most deadly gynecologic malignancy. ${ }^{1}$ Although more than $60 \%$ of cases are diagnosed in advanced stages (stages III and IV), stage I represents one quarter of all new epithelial ovarian cancer diagnoses, and the overall 5-year survival is reported to be between $70 \%$ and $89 \% .^{2-4}$ Epithelial ovarian cancer staging is surgical and is based on the 1988 International Federation of Gynecology and Obstetrics (FIGO) staging criteria. ${ }^{5}$ Stage IA encompasses all unilateral, unruptured ovarian cancers without surface involvement. Stage IB epithelial ovarian cancers are bilateral, unruptured, and without surface involvement. Epithelial ovarian cancers confined to the ovary are assigned to stage IC if there is evidence of capsule surface involvement or 
positive cytology in peritoneal washings obtained at time of exploration. In addition, if the capsule of a stage IA or IB cancer is ruptured either preoperatively or intraoperatively, the patient is upstaged to stage IC. When patients are managed only with surgery, the overall 5-year survival for stage IA approaches 94\% and stage IB 92\%, whereas 5-year survival for stage IC is $84 \%$ in some series. ${ }^{2}$ For stage I epithelial ovarian cancer overall, the 5 -year recurrence-free survival after appropriate staging and adjuvant chemotherapy approaches $85 \%{ }^{6}$

The standard of care today for management of epithelial ovarian cancer includes surgical staging and tumor cytoreduction when indicated, followed by adjuvant platinum-based chemotherapy. ${ }^{7}$ This approach has yielded improved disease-free survival and overall survival. ${ }^{8-11}$ Factors that delineate which early-stage patients are at highest risk for recurrence are used to determine whether to administer adjuvant chemotherapy. Important prognostic factors include grade, histologic subtype, and substage. ${ }^{12}$ Optimal adjuvant treatment of high-risk early stage epithelial ovarian cancer continues to be debated (Markman M. Re: "Randomized phase III trial of three compared with six cycles of adjuvant carboplatin and paclitaxel in early stage epithelial ovarian carcinoma: a Gynecologic Oncology Group study" [letter]. Gynecol Oncol 2007;105: $279-80) .^{3}$ However, it is generally agreed that after comprehensive staging, well-differentiated or moderately differentiated stage IA cancers do not benefit from adjuvant chemotherapy, because their overall prognosis is excellent without adjuvant treatment. ${ }^{9,12,13,14}$ In addition, per the National Comprehensive Center Network guidelines, both comprehensively staged grade 1 and 2, stage IA and IB epithelial ovarian cancer can be observed. ${ }^{15}$ For all other categories of stage I disease, adjuvant treatment is recommended.

One area of conflict is the prognostic significance of capsule rupture during surgery in a case that would otherwise be assigned to stage IA or IB. Several studies have suggested that intraoperative rupture of an intact capsule does not affect prognosis, $, 216,17$ whereas others indicate intraoperative rupture is an independent predictor of decreased disease-free survival ${ }^{18-21}$ and worse overall survival. ${ }^{19}$ Unfortunately, most of these studies are limited by inclusion of nonstaged or incompletely staged cases, lack of consistent adjuvant treatments, and lack of subset analyses of categories within stage IC tumors. These limitations make interpretation of the existing literature fraught with errors. We designed this retrospective study to investigate the effect of intraoperative capsule rupture on disease-free survival and disease-specific survival in patients with stage I epithelial ovarian cancer who were completely staged and all treated during the platinum chemotherapy era according to similar standards.

\section{MATERIALS AND METHODS}

After institutional review board approval at both institutions, all cases of stage I epithelial ovarian cancer were identified that had their initial diagnoses and staging procedures either at The Mayo Clinic or at The Ohio State University College of Medicine between January 1991 and December 2007. Medical records were reviewed and data abstracted, including demographics, surgical stage, tumor grade, operative staging procedures, intraoperative findings, adjuvant therapies, and follow-up, including recurrence and death events. Inclusion criteria included complete records, comprehensive staging, staging operation performed at either the Mayo Clinic or The Ohio State University, and patient consent for review of their medical records. Comprehensive surgical staging was defined as exploratory laparotomy, collection of cytologic washings, hysterectomy (if uterus present), bilateral salpingo-oophorectomy, bilateral pelvic and paraaortic lymphadenectomy, omentectomy, and peritoneal biopsies. Patients were excluded from the analysis if they did not undergo one or more of the staging procedures. Patients were excluded if they were referred for staging after the diagnosis was made elsewhere or distant in time from the diagnostic operative procedure or if they had preoperative drainage or cyst aspiration. All cases were either independently reviewed by a single gynecologic pathologist (Mayo Clinic) or re-reviewed by a gynecologic pathologist at tumor board as part of clinical decision making (The Ohio State University College of Medicine).

For the purpose of identifying and separating the various indications for assignment to stage IC, we defined substages of stage IC as follows: ICr-patients who had intraoperative capsule rupture as their only stage IC qualification (eg, no surface involvement and negative cytology); ICs/w, no rupture-patients with surface excrescences and/or positive cytologic washings whose tumors were removed intact; ICs/w, with rupture-patients with surface excrescences and/or positive cytologic washings whose tumors also ruptured intraoperatively.

The primary outcomes chosen were disease-free survival and disease-specific survival. Disease-specific survival was chosen instead of overall survival because many patients with early stage disease who are rendered disease free will die from other causes without recur- 
rence. Cox proportional hazards, Kaplan-Meier estimation, $\log$ rank test, and $\chi^{2}$ test were used for statistical analyses. Stepwise multivariable analysis was performed to determine factors significantly affecting disease-free survival. Due to the small number of patients who died from disease, multivariable analysis of factors affecting disease-specific survival was not performed. The JMP 7.0 (SAS Institute Inc., Cary, NC) statistical program was used for all analyses.

\section{RESULTS}

During the study interval, there were 161 patients diagnosed with surgical stage I epithelial ovarian cancer who underwent comprehensive surgical staging. All patients underwent immediate comprehensive surgical staging after intraoperative frozen section determination of cancer.

Substaging was as follows: $65(40 \%)$ FIGO stage IA, $9(6 \%)$ IB, and $87(54 \%)$ IC. Thirty-eight $(44 \%)$ of the stage IC cases would have been considered stage IA or IB if the tumor capsule had remained intact. Overall, for the 87 cases of IC disease, the reasons for upstaging were as follows: $38(44 \%)$ secondary to intraoperative capsule rupture only ( $\mathrm{ICr}) ; 26(30 \%)$ due to ovarian surface involvement with tumor and/or positive cytology from washings and no intraoperative rupture of the capsule (ICs/w, no rupture); and $23(26 \%)$ due to ovarian surface involvement with tumor and/or positive cytology from washings in addition to intraoperative rupture of the capsule (ICs/w, with rupture) (Table 1).

Overall, 22 patients (14\%) developed recurrent disease, and 12 patients $(7 \%)$ died of disease. The median follow-up among the 139 patients alive at the time of last follow-up was 44.7 months (range 0.2-185 months, mean 54.1 months). Mean \pm standard deviation pelvic and paraaortic lymph node counts were $26.8 \pm 13.8$ and $11.7 \pm 7.2$, respectively.

In univariable analysis, capsule rupture portended a lower disease-free survival (hazard ratio [HR] 3.6, 95\% confidence interval [CI] 1.5-8.9; $P=.004$ ) (Table 2) and a lower disease-specific survival (HR 4.1, 95\% CI 1.3-15.4; $P=.018$ ) (Table 3). When controlling for positive cytology, intraoperative capsule rupture remained a predictor of poorer disease-free survival (HR 4.2, 95\% CI 1.8-10.9; $P=.001$ ).

There was similar disease-free survival $(P=.84$, $\log$ rank $)$ and disease-specific survival $(P=.97, \log$ rank) among patients who were assigned to stage IC due to a single factor alone (eg, only positive cytology or surface involvement or capsule rupture). Among all stage IC cases, the shortest disease-free survival and disease-specific survival were observed in pa-
Table 1. Stage I Epithelial Ovarian Cancer Patient Characteristics, Substage, Tumor Characteristics, and Adjuvant Therapy

\begin{tabular}{|c|c|}
\hline Characteristics & $\begin{array}{l}\text { Patients } \\
(n=161)\end{array}$ \\
\hline \multicolumn{2}{|l|}{ Age $(y)$} \\
\hline Mean (range) & $56(23-83)$ \\
\hline Median & 58 \\
\hline \multicolumn{2}{|l|}{ Substage } \\
\hline IA & $65(40)$ \\
\hline IB & $9(6)$ \\
\hline IC & $87(54)$ \\
\hline $\begin{array}{l}\text { Intraoperative rupture only } \\
(\mathrm{ICr})^{*}\end{array}$ & $38(44)$ \\
\hline $\begin{array}{l}\text { Surface involvement and/or } \\
\text { positive cytology without } \\
\text { capsule rupture (ICs/w, no } \\
\text { rupture) })^{\dagger}\end{array}$ & $26(30)$ \\
\hline $\begin{array}{l}\text { Surface involvement and/or } \\
\text { positive cytology and } \\
\text { capsule rupture (ICs/w, with } \\
\text { rupture })^{\ddagger}\end{array}$ & $23(26)$ \\
\hline \multicolumn{2}{|l|}{$\begin{array}{l}\text { Individual stage IC factors (among } \\
\text { all stage IC patients) }\end{array}$} \\
\hline $\begin{array}{l}\text { Intraoperative capsule rupture } \\
\text { occurred }\end{array}$ & $61(38)$ \\
\hline Positive cytology & $33(20)$ \\
\hline Surface involvement present & $22(14)$ \\
\hline \multicolumn{2}{|l|}{ Grade } \\
\hline 1 & $34(21)$ \\
\hline 2 & $45(28)$ \\
\hline 3 & $82(51)$ \\
\hline \multicolumn{2}{|l|}{ Tumor histology } \\
\hline Serous & $29(18)$ \\
\hline Clear cell & $35(22)$ \\
\hline Mucinous & $27(17)$ \\
\hline Endometrioid & $59(37)$ \\
\hline Other ${ }^{\S}$ & $11(6)$ \\
\hline \multicolumn{2}{|l|}{ Tumor mobility } \\
\hline Mobile & $74(46)$ \\
\hline Fixed & $77(48)$ \\
\hline Not recorded & $10(6)$ \\
\hline \multicolumn{2}{|l|}{$\begin{array}{l}\text { Platinum-based chemotherapy } \\
\text { received }\end{array}$} \\
\hline Yes & $106(66)$ \\
\hline No & $55(34)$ \\
\hline
\end{tabular}

Data are $\mathrm{n}(\%)$ except where otherwise specified.

* Based on operative note; intraoperative rupture as only indication for stage IC.

$\uparrow$ Surface involvement based on pathology findings and/or positive cytology from washings, no capsule rupture.

¥ Surface involvement based on pathology findings and/or positive cytology from washings, and capsule rupture.

$\S$ Mixed, transitional cell, and seroanaplastic.

tients who had a combination of positive cytology and/or surface involvement along with intraoperative capsule rupture (ICs/w, with rupture). Patients classified as ICs/w, with rupture had significantly worse disease-free survival (HR 3.3, 95\% CI 1.1-12.1; 
Table 2. Univariable Analysis of Factors Important in Disease-Free Survival

\begin{tabular}{llr}
\hline Variable & HR $(\mathbf{9 5} \% \mathrm{CI})$ & \multicolumn{1}{c}{$\boldsymbol{P}$} \\
\hline Capsule rupture (yes) & $3.6(1.5-8.9)$ & .004 \\
Cytology (positive) & $5.2(2.1-12.3)$ & $<.001$ \\
Surface involvement (yes) & $2.6(0.9-6.4)$ & .064 \\
Grade (high) & $2.3(1.0-5.9)$ & .064 \\
Tumor mobility (fixed) & $1.6(0.7-4.4)$ & .29 \\
Received platinum-based & $1.7(0.6-6.0)$ & .31 \\
$\quad$ chemotherapy (yes) & & \\
Stage $(1 \mathrm{C}$ vs 1A/B) & $5.0(1.9-17.3)$ & $<.001$ \\
\hline
\end{tabular}

HR, hazard ratio; CI, confidence interval.

$P=.041)$ compared with $\mathrm{ICs} / \mathrm{w}$, no rupture patients. Disease-specific survival was not significantly different between these two cohorts; however, there were only three (of 26) ICs/w, no rupture patients and five (of 23) ICs/w, with rupture patients who died from disease, thus limiting power. Patients assigned to IC solely due to rupture (ICr) had outcomes similar to those assigned to IC due to surface involvement and/or positive cytology (ICs/w, no rupture) (Fig. 1).

There were $38(44 \%)$ stage IC patients who were upstaged to IC from IA/B disease based solely on intraoperative capsule rupture (ICr). These patients all had negative cytologic washings before tumor manipulation as well as no ovarian surface excrescences on final pathology. There were five recurrences $(13.2 \%)$ and three deaths from disease $(7.9 \%)$ among the $38 \mathrm{ICr}$ patients; there were four recurrences $(5.4 \%)$ and only one death from disease (1.4\%) among the $74 \mathrm{IA} / \mathrm{B}$ patients. In time-to-event analyses, differences in disease-free survival (HR 2.7, 95\% CI $0.7-10.9 ; P=.14$ ) (Fig. 2) and disease-specific survival (HR 5.3, 95\% CI 0.7-107.8; $P=.12$ ) between stages IA/B and ICr were observed but they were not statistically significant. This also likely reflects the limited power due to the small number of events. Overall, disease-free survival was shorter (HR 5.0, $95 \%$ CI $1.9-17.3 ; \quad P<.001)$, and disease-specific

Table 3. Univariable Analysis of Factors Important in Disease-Specific Survival

\begin{tabular}{lll}
\hline Variable & HR $(95 \% \mathrm{CI})$ & $\boldsymbol{P}$ \\
\hline Capsule rupture (yes) & $4.1(1.3-15.4)$ & .018 \\
Cytology (positive) & $5.9(1.8-19.3)$ & .005 \\
Surface involvement (yes) & $2.4(0.5-8.4)$ & .23 \\
Grade (high) & $5.5(1.4-36.1)$ & .011 \\
Tumor mobility (fixed) & $1.2(0.4-4.2)$ & .77 \\
Received platinum-based & $4.0(0.8-74.3)$ & .11 \\
$\quad$ chemotherapy (yes) & & \\
Stage (1C vs 1A/B) & $10.6(2.0-193.8)$ & .002 \\
\hline
\end{tabular}

$\mathrm{HR}$, hazard ratio; CI, confidence interval.

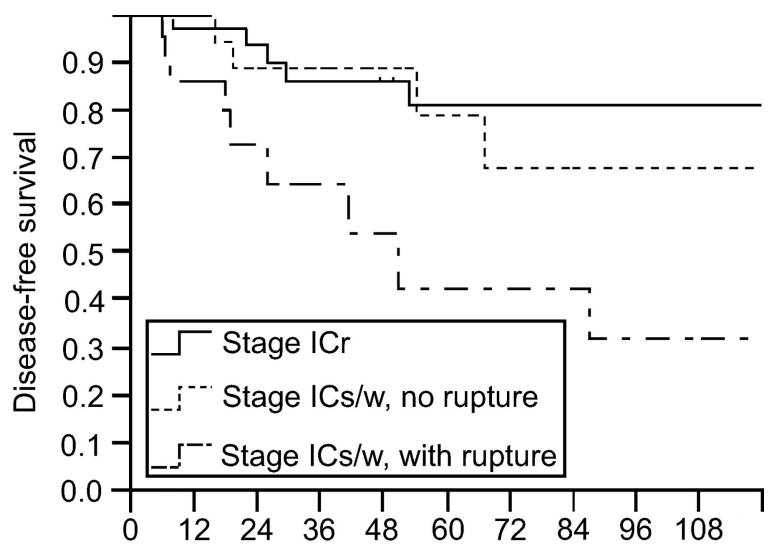

A Time to recurrence (months)

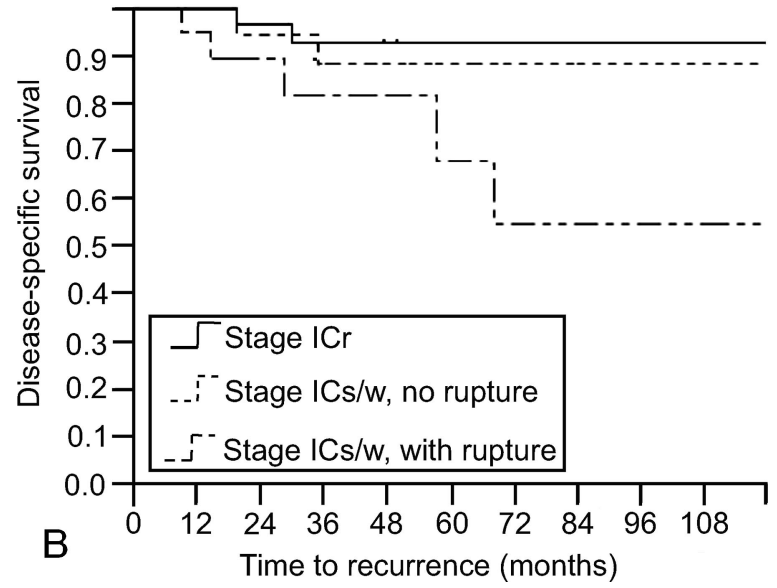

Fig. 1. Subclassification of stage IC and effect on $\mathbf{A}$. disease-free survival $(P=.008, \log$ rank test) and $\mathbf{B}$. diseasespecific survival $(P=.042, \log$ rank test). ICr, stage IC, secondary to capsule rupture only. ICs/w, no rupturepatients with surface excrescences and/or positive cytologic washings whose tumors were removed intact; ICs/w, with rupture-patients with surface excrescences and/or positive cytologic washings whose tumors also ruptured intraoperatively.

Bakkum-Gamez. Intraoperative Capsule Rupture. Obstet Gynecol 2009.

survival was shorter (HR 10.6, 95\% CI 2.0-193.8; $P=.002)$, for combined stage IC cases compared with stage IA/B cases.

Clear descriptions of the ovarian tumors' intraoperative mobility were available for 151 of $161(94 \%)$ cases such that we could accurately classify them as either fixed or mobile. Nearly an equal percentage of tumors were described as mobile $(46 \%)$ as were described as fixed $(48 \%)$ in the operative notes (Table 1). There was no correlation between tumor mobility and surface involvement of tumor $\left(P=.36, \chi^{2}\right)$ or positive cytology $\left(P=.47, \chi^{2}\right)$. There was a higher rate of capsule rupture when tumors were described by 


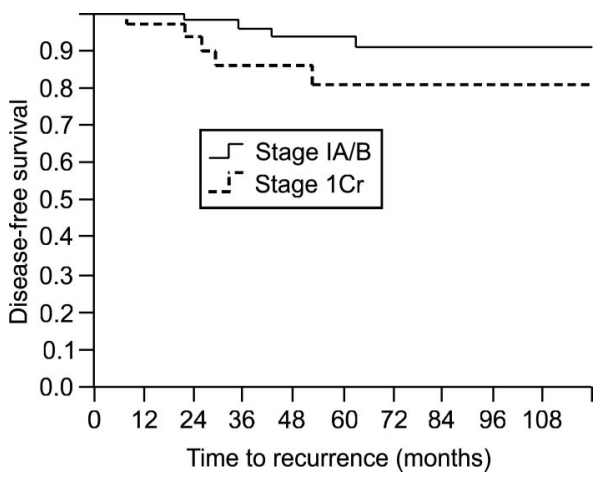

Fig. 2. Disease-free survival in stage $I A / B$ and stage $I C$ secondary to capsule rupture only $(\mathrm{ICr}) ;(P=.12$, log-rank test). ICr, stage IC, secondary to capsule rupture only.

Bakkum-Gamez. Intraoperative Capsule Rupture. Obstet Gynecol 2009.

the surgeon as fixed $\left(P=.012, \chi^{2}\right)$. There was not a significant difference in tumor mobility when comparing stages IA/B to stage $\operatorname{ICr}\left(P=.094, \chi^{2}\right)$, but there was a higher rate of tumor fixation for ICs/w, with rupture compared with ICs/w, no rupture $(P=.045$, $\left.\chi^{2}\right)$. Overall, the finding of a fixed mass did not affect disease-free survival (HR 1.6, 95\% CI 0.7-4.4; $P=.29$ ) (Table 2) or disease-specific survival (HR 1.2; 95\% CI $0.4-4.2 ; P=.77$ ) (Table 3).

In univariable analyses, having positive cytologic washings decreased disease-free survival (HR 5.2, 95\% CI 2.1-12.3; $P<.001)$ and disease-specific survival (HR 5.9, 95\% CI 1.8-19.3; $P=.005$ ), whereas having ovarian surface excrescences alone had no effect on disease-free survival or disease-specific survival (Table 2 and 3). Controlling for intraoperative capsule rupture, the presence of positive cytology remained a predictor of worse disease-free survival (HR 6.4, 95\% CI 2.5-16.0; $P<.001$ ).

Twenty-six patients were stage IC based on surface involvement and/or positive cytologic washings but had their tumors removed intact without rupture. Fifteen $(58 \%)$ had only positive cytology and no evidence of surface involvement, eight (31\%) had surface involvement only and negative cytology, and three $(11 \%)$ had both positive cytology and surface involvement. Twenty-three additional patients were stage IC based on surface involvement and/or positive cytologic washings and also sustained capsule rupture during surgery. Twelve $(52 \%)$ had positive cytology and tumor rupture; eight $(35 \%)$ had surface involvement and tumor rupture; and three (13\%) had both positive cytology and surface involvement in addition to tumor rupture.

In univariable analysis, receiving platinum-based chemotherapy was not associated with worse disease- specific survival or disease-free survival (Table 2 and 3). Overall, 106 of 161 (66\%) patients received platinumbased chemotherapy, and most (91 of 106) received intravenous carboplatin and paclitaxel. Administration of chemotherapy was stage-dependent, being most common for stage IC cases ( 74 of $87,85 \%)$. Stage IC patients were more likely to receive adjuvant treatment than stage IA/B, because only $43 \%$ (32 of 74 ) of the stage IA/B cases received platinum-based chemotherapy $\left(P<.001, \chi^{2}\right)$. Chemotherapy was administered in substages of IC as follows: Icr-34 of 38 cases $(89 \%) ; \mathrm{ICs} / \mathrm{w}$, no rupture-18 of 26 cases $(69 \%)$; ICs $/ w$, with rupture-22 of 23 cases $(96 \%)$. There was a significant difference in the percentage of patients receiving chemotherapy among all substages.

Thirteen stage IC patients did not receive platinum-based chemotherapy. Reasons for not receiving platinum-based chemotherapy among stage IC patients were as follows: stage Icr-one patient refused and two patients did not receive chemotherapy for reasons unknown; stage $\mathrm{ICs} / \mathrm{w}$, no rupture-one received intraperitoneal P32, one had recurrent melanoma, one died on postoperative day 18 from a myocardial infarction, and one did not receive chemotherapy for reasons unknown; and stage ICs/w, with rupture-1 received intraperitoneal P32. Intraperitoneal P32 was not considered equal to platinumbased chemotherapy. ${ }^{22-24}$ An additional eight patients received follow-up at outside institutions and receipt of chemotherapy is unknown. Three were stage IA/B, one was stage ICr, and four were stage $\mathrm{ICs} / \mathrm{w}$, no rupture.

Despite adjuvant treatment, $4(12 \%)$ of the $34 \mathrm{ICr}$ patients who received chemotherapy developed disease recurrence, and three died from their disease. In addition, 17\% (3 of 18) of stage ICs/w, no rupture cases that received chemotherapy and 36\% (8 of 22) stage $\mathrm{ICs} / \mathrm{w}$, with rupture cases that received chemotherapy developed disease recurrence. Fifteen percent (6 of 40) of stage ICs/w cases overall (those with capsule rupture and those without) who received chemotherapy died of their disease. Nine percent (3 of 34) of ICr cases and only $3 \%$ (1 of 32 ) of IA/B cases who received platinum-based chemotherapy died of disease.

Just over one half of the epithelial ovarian cancers were FIGO grade 3 (Table 1). We dichotomized tumors as either low-grade (FIGO grade 1 or 2) or high-grade (FIGO grade 3) for analyses. In univariable analysis, higher grade demonstrated a tendency to affect disease-free survival but did not reach statistical significance (HR 2.3, 95\% CI 1.0-5.9; $P=.064$ ). Higher grade did portend a worse disease-specific survival (HR 5.5, 95\% CI 1.4-36.1; $P=.011$ ) (Table 2). 
Additionally, there was a higher likelihood of receiving platinum-based chemotherapy associated with highgrade tumor $\left(P<.001, \chi^{2}\right)$. The most common histology was endometrioid (37\%), followed by clear cell $(22 \%)$, serous $(18 \%)$, mucinous (17\%), and other (mixed, transitional cell, and seroanaplastic) histologies (6\%). There was no difference in disease-free survival or diseasespecific survival based on histologic subtype.

\section{DISCUSSION}

The effects of intraoperative capsular rupture on outcomes in stage I epithelial ovarian cancer have been difficult to elucidate due to the conflicting published literature and the heterogenous populations of patients included in these reports leading to flawed conclusions. Most notably, prior publications have included, or been comprised of, a large percentage of incompletely staged patients, ${ }^{2,10,16,18,19,21}$ patients who received non-platinum-based chemotherapy ${ }^{19}$ or radiation as their adjuvant therapy, ${ }^{21}$ and the inclusion of borderline tumors. ${ }^{16}$ The cohort of women that we studied was highly homogeneous, because all of them had invasive epithelial ovarian cancer, underwent complete staging procedures, and if adjuvant therapy was administered, it was a platinum-based regimen.

In this retrospective, two-institution study, we analyzed the importance of intraoperative tumor rupture on prognosis in a uniform cohort of early stage epithelial ovarian cancer. We observed both a shorter time to disease recurrence and shorter disease-specific survival associated with intraoperative capsule rupture both alone and when controlling for positive cytology, which was also an independent predictor of worse disease-free survival and disease-specific survival. While positive cytology was a significant predictor of worse prognosis, washings were collected before tumor manipulation and capsule rupture (if rupture occurred) suggesting that malignant cells within the pelvis and abdomen are an inherent finding at the time of surgery for some patients. Capsule rupture, however, may be preventable with careful surgical technique.

We noted a clinically significant difference in disease-free survival and disease-specific survival between stage IA/B and stage ICr patients; however, this difference did not reach statistical significance (Figure 2). We attribute this to the small number of recurrences and deaths from disease in both groups and while analysis of a larger number of IA/B and ICr patients is warranted, this factor should be considered when planning adjuvant therapy. The worst diseasefree survival and disease-specific survival were observed in patients who had tumor rupture in addition to surface excrescences and/or malignant cells present in their pelvic washings. Thus, it seems that intraoperative capsule rupture further worsens the prognosis of those women who are already stage IC based on the unalterable findings of surface involvement and/or positive cytology.

One of the strengths of our study is the restriction to cases that were comprehensively staged, including pelvic washings, hysterectomy, bilateral salpingo-oophorectomy, omentectomy, pelvic and paraaortic lymphadenectomy, and peritoneal biopsies. An additional strength of the study is that all patients treated with intravenous chemotherapy received platinumbased regimens. Despite our restrictions, our findings are consistent with the most recent published data which also suggests there is a worse prognosis for stage I patients who have intraoperative capsule rupture. ${ }^{18-21}$ A limitation in any retrospective surgical series is the dependence upon operative notes for intraoperative factors, such as the presence of peritumoral adhesions, which may also contribute to intraoperative rupture. In addition, when comparing stage IA/B cases to stage $\mathrm{ICr}$ cases, we lacked the power to show a statistically significant difference in diseasefree survival and disease-specific survival despite the HR of 2.7 and 5.3, respectively.

When considering why intraoperative capsular rupture might be an important prognostic factor, we developed several hypothesis-driven questions. First, is tumor fixation the true prognostic indicator and the capsule rupturing during removal simply a surrogate of tumor immobility? Although fixed tumors were more likely to rupture, neither recurrence nor death from disease were associated with mass immobility. Importantly, we also noted that tumor immobility did not correlate with surface involvement on final pathology. Second, are we seeing a prognostic effect of lag time between diagnosis (ie, laparoscopic salpingo-oophorectomy) and definitive staging? In this study, no patients were included whose tumors were removed for diagnosis and referred weeks later for definitive surgical management. All of the women in this study underwent immediate staging by a gynecologic oncologist and irrigation of the peritoneal cavity at the time of frozen-section diagnosis. This eliminates lag time as a factor.

We are left suggesting two reasonable concepts for why intraoperative rupture may affect outcomes. First, that intraoperative spread of viable tumor occurs at the time of rupture. This suggests very careful avoidance of rupture is warranted, and if it occurs, immediate and copious irrigation should be performed, perhaps before completion of staging operation to avoid seeding retroperitoneal spaces and then again at the end of surgery. A second reasonable 
explanation is that occult invasion by cancer of the capsular surface exists, which predisposes to rupture at that site intraoperatively. After rupture it may be more difficult to histologically identify microscopic surface involvement at the rupture site. And thus, rupture is merely reflective of innately more aggressive tumor biology. Gene expression studies comparing unruptured compared with ruptured cases may lend useful information on the basic biologic properties of these two clearly distinct types of cancers.

Overall, this study supports previous findings that intraoperative capsule rupture leads to a worse disease prognosis. This should be considered when counseling patients and considering adjuvant therapy, particularly when considering duration of treatment, because many authors are now suggesting three cycles of adjuvant chemotherapy may be sufficient for early stage disease. $^{3,25}$ Our data suggest that current treatment regimens leave patients at high risk of recurrence and death within 5 years for stage I cases with surface involvement and/or positive cytology and tumor rupture. This should serve as reminder to both patient and oncologist of the significance of this disease even in early stage cases. Obviously, careful removal of an intact cystic mass, even in the setting of adhesions, should be the goal in the surgical management of stage I epithelial ovarian cancer.

\section{REFERENCES}

1. Jemal A, Siegel R, Ward E, Hao Y, Xu J, Murray T, et al. Cancer statistics, 2008. CA Cancer J Clin 2008;58:71-96.

2. Ahmed FY, Wiltshaw E, A'Hern RP, Nicol B, Shepherd J, Blake P, et al. Natural history and prognosis of untreated stage I epithelial ovarian carcinoma. J Clin Oncol 1996;14:2968-75.

3. Bell J, Brady MF, Young RC, Lage J, Walker JL, Look KY, et al. Randomized phase III trial of three versus six cycles of adjuvant carboplatin and paclitaxel in early stage epithelial ovarian carcinoma: a Gynecologic Oncology Group study. Gynecol Oncol 2006;102:432-9.

4. Nguyen HN, Averette HE, Hoskins W, Sevin BU, Penalver M, Steren A. National survey of ovarian carcinoma. VI. Critical assessment of current International Federation of Gynecology and Obstetrics staging system. Cancer 1993;72:3007-11.

5. Makar AP, Trope C. Fertility preservation in gynecologic cancer. Acta Obstet Gynecol Scand 2001;80:794-802.

6. Skirnisdottir I, Sorbe B. Survival and prognostic factors in earlystage epithelial ovarian carcinoma treated with taxane-based adjuvant chemotherapy. Int J Gynecol Cancer 2007;17:1231-7.

7. Aletti GD, Gallenberg MM, Cliby WA, Jatoi A, Hartmann LC. Current management strategies for ovarian cancer. Mayo Clin Proc 2007;82:751-70.

8. Aletti GD, Dowdy SC, Gostout BS, Jones MB, Stanhope CR, Wilson TO, et al. Aggressive surgical effort and improved survival in advanced-stage ovarian cancer. Obstet Gynecol 2006;107: 77-85.

9. Colombo N, Guthrie D, Chiari S, Parmar M, Qian W, Swart $\mathrm{AM}$, et al. International Collaborative Ovarian Neoplasm trial 1: a randomized trial of adjuvant chemotherapy in women with early-stage ovarian cancer. J Natl Cancer Inst 2003;95:125-32.
10. Trimbos JB, Parmar M, Vergote I, Guthrie D, Bolis G, Colombo N, et al. International Collaborative Ovarian Neoplasm Trial 1 and Adjuvant ChemoTherapy In Ovarian Neoplasm Trial: two parallel randomized phase III trials of adjuvant chemotherapy in patients with early-stage ovarian carcinoma. J Natl Cancer Inst 2003;95:105-12.

11. Winter WE 3rd, Maxwell GL, Tian C, Carlson JW, Ozols RF, Rose PG, et al. Prognostic factors for stage III epithelial ovarian cancer: a Gynecologic Oncology Group Study. J Clin Oncol 2007;25:3621-7.

12. Trope C, Kaern J. Adjuvant chemotherapy for early-stage ovarian cancer: review of the literature. J Clin Oncol 2007;25:2909-20.

13. Borgfeldt C, Iosif C, Masback A. Fertility-sparing surgery and outcome in fertile women with ovarian borderline tumors and epithelial invasive ovarian cancer. Eur J Obstet Gynecol Reprod Biol 2007;134:110-4.

14. Trimbos JB, Vergote I, Bolis G, Vermorken JB, Mangioni C, Madronal C, et al. Impact of adjuvant chemotherapy and surgical staging in early-stage ovarian carcinoma: European Organisation for Research and Treatment of Cancer-Adjuvant ChemoTherapy in Ovarian Neoplasm trial. J Natl Cancer Inst 2003;95:113-25.

15. National Comprehensive Cancer Network. Ovarian Cancer, v. 1.2008. Available at: www.nccn.org/professionals/physician_gls/ PDF/ovarian.pdf . Retrieved October 21, 2008.

16. Dembo AJ, Davy M, Stenwig AE, Berle EJ, Bush RS, Kjorstad K. Prognostic factors in patients with stage I epithelial ovarian cancer. Obstet Gynecol 1990;75:263-73.

17. Kodama S, Tanaka K, Tokunaga A, Sudo N, Takahashi T, Matsui $\mathrm{K}$. Multivariate analysis of prognostic factors in patients with ovarian cancer stage I and II. Int J Gynaecol Obstet 1997;56: $147-53$.

18. Mizuno M, Kikkawa F, Shibata K, Kajiyama H, Suzuki T, Ino $\mathrm{K}$, et al. Long-term prognosis of stage I ovarian carcinoma. Prognosis importance of intraoperative rupture. Oncology 2003;65:29-36.

19. Sainz de la Cuesta R, Goff BA, Fuller AF Jr, Nikrui N, Eichhorn JH, Rice LW. Prognostic importance of intraoperative rupture of malignant ovarian epithelial neoplasms. Obstet Gynecol 1994;84:1-7.

20. Vergote I. Prognostic factors in stage I ovarian carcinoma Verh K Acad Geneeskd Belg 2001;63:257-71.

21. Vergote I, De Brabanter J, Fyles A, Bertelsen K, Einhorn N, Sevelda $\mathrm{P}$, et al. Prognostic importance of degree of differentiation and cyst rupture in stage I invasive epithelial ovarian carcinoma. Lancet 2001;357:176-82.

22. Bolis G, Colombo N, Pecorelli S, Torri V, Marsoni C, Bonazzi $\mathrm{C}$, et al. Adjuvant treatment for early epithelial ovarian cancer: results of two randomised clinical trials comparing cisplatin to no further treatment or chromic phosphate (32P). G.I.C.O.G.: Gruppo Interregionale Collaborative in Ginecologia Oncologica. Ann Oncol 1995;6:887-93.

23. Soper JT, Berchuck A, Dodge R, Clarke-Pearson DL. Adjuvant therapy with intraperitoneal chromic phosphate $(32 \mathrm{P})$ in women with early ovarian carcinoma after comprehensive surgical staging. Obstet Gynecol 1992;79:993-7.

24. Young RC, Brady MF, Nieberg RK, Long HJ, Mayer AR, Lentz SS, et al. Adjuvant treatment for early ovarian cancer: a randomized phase III trial of intraperitoneal $32 \mathrm{P}$ or intravenous cyclophosphamide and cisplatin-a gynecologic oncology group study. J Clin Oncol 2003;21:4350-5.

25. Shimada M, Kigawa J, Kanamori Y, Itamochi H, Oishi T, Minagawa Y, et al. Outcome of patients with early ovarian cancer undergoing three courses of adjuvant chemotherapy following complete surgical staging. Int J Gynecol Cancer 2005;15:601-5. 